\title{
Meteorología y resultados electorales en las elecciones locales españolas
}

\author{
Joaquín Artés Caselles \\ Universidad Complutense de Madrid
}

\section{Resumen}

Este artículo analiza el efecto de las condiciones meteorológicas adversas en el nivel de participación en las elecciones locales españolas. Los resultados muestran que la meteorología adversa es un coste para el votante que se traduce en un menor nivel de participación. En concreto, la participación electoral es aproximadamente medio punto porcentual menor en aquellos municipios en los que llueve respecto a aquellos en los que no llueve en el día de las elecciones. Los resultados muestran también que la meteorología no afecta por igual a todos los partidos, siendo los partidos conservadores los más beneficiados por la presencia de lluvia.

Palabras clave: elecciones, abstención, lluvia.

Clasificación JEL: D72.

\begin{abstract}
This paper analyzes the effect of adverse weather conditions on voting participation in Spanish local elections. I find that inclement weather is a cost imposed on voting that reduces electoral turnout. In particular, I find that turnout is approximately half a percentage point lower in municipalities that experienced bad weather on election day. Additionally, I find that inclement weather has partisan effects, being the conservative parties the ones benefited by it.
\end{abstract}

Keywords: elections, turnout, rain.

JEL classification: D72.

\section{Introducción}

En este artículo se analiza el efecto de la meteorología adversa en el nivel de participación electoral en España. También se analiza si la meteorología adversa afecta más a los votantes de unos partidos políticos u otros. Tal y como ponen de manifiesto Gómez, Hansford y Krause (2007), estas cuestiones resultan de interés puesto que aunque apenas existen datos o literatura científica sobre el tema, parece existir la creencia generalizada de que la meteorología adversa en el día de las elecciones reduce la participación electoral y perjudica a los partidos de izquierdas en mayor medida que a los de derechas.

Para dar una idea del arraigo de la creencia de que los resultados electorales se pueden ver afectados por las malas condiciones meteorológicas, basta acudir a los medios de comunicación. Un reciente artículo de opinión publicado en el Washington Post unos días antes de las elecciones americanas de 2012 sugería que aproximadamente un tercio de votantes declaraban tener una probabilidad alta o muy 
alta de no acudir a las urnas si llovía o nevaba el día de las elecciones ${ }^{1}$. Además, la mayor parte de los votantes que manifestaban que la meteorología adversa afectaría a su probabilidad de acudir a las urnas se declaraban votantes de Obama, y afirmaban ser de renta media o baja. De igual modo, el Corriere della Sera, uno de los principales periódicos italianos, publicaba unos días antes de las elecciones italianas de 2013 un artículo de opinión en el que se afirmaba que la climatología adversa tendría un papel decisivo el día de las elecciones, beneficiando a Beppe Grillo y perjudicando a los candidatos de los partidos tradicionales (Berlusconi y Barsani) ${ }^{2}$. Ese artículo apoyaba su razonamiento en la idea de que los votantes de mayor edad y los votantes indecisos serían los más afectados por las malas condiciones meteorológicas, y apoyarían, en caso de acudir a las urnas, a los candidatos de los partidos tradicionales. En España, un artículo publicado en el diario El Mundo unos días antes de las elecciones de 2011 afirmaba que la lluvia podía retraer a un gran número de votantes, y llegaba aún más lejos al afirmar que podría dar lugar a cambios de estado anímico que podrían incluso cambiar el sentido del voto de algunos votantes ${ }^{3}$.

Ejemplos como los anteriores pueden encontrarse en un gran número de medios de comunicación y para distintos países, y ponen de manifiesto el arraigo de la creencia de que las condiciones meteorológicas son susceptibles de afectar a los resultados electorales. Sin embargo, ninguno de estos artículos de opinión citaba o se apoyaba en estudio científico alguno, sino que recogían simplemente la opinión del periodista o de los ciudadanos al respecto. El presente trabajo tiene como objetivo aportar evidencia empírica sobre si efectivamente la meteorología adversa tiene efectos en los resultados electorales. Se pretende cuantificar el impacto y además comprobar si existen diferencias entre los distintos partidos políticos en los efectos producidos. Para contestar a estas preguntas se analizarán datos electorales a nivel de municipio correspondientes a las siete elecciones locales celebradas en España entre 1987 y 2011, y se enlazarán con datos sobre nivel de precipitación en cada municipio en el día de las elecciones proporcionados por la Agencia Estatal de Meteorología (AEMET).

Para poder comprender adecuadamente los resultados del trabajo, en la siguiente sección se discuten las hipótesis teóricas que se contrastan y se lleva a cabo una revisión de la literatura científica sobre la relación entre meteorología y resultados electorales. A continuación se formula el modelo econométrico correspondiente, se describen los datos utilizados y se discuten los resultados obtenidos. Finalmente, el último apartado del trabajo se dedica a resaltar las principales conclusiones alcanzadas.

${ }^{1}$ El artículo fue publicado el 5 de noviembre de 2012 en la versión on-line del Washington Post con el título «Will weather affect Election Day results for President and Congress?». Un artículo de temática similar fue publicado en ese mismo periódico para las elecciones de 2008 el 30 de octubre de 2008 con el título «Weathering the Election».

${ }^{2}$ El artículo en inglés puede consultarse en la edición on-line de The Telegraph con el título «Italy Election hampered by bad weather as Beppe Grillo looms», publicado el 24 de febrero de 2013.

${ }^{3}$ El artículo fue publicado el 19 de noviembre de 2011 con el título «La lluvia afecta al estado anímico y puede determinar el sentido del voto». 


\section{Literatura e hipótesis}

Desde el punto de vista teórico las razones por las que la meteorología adversa es susceptible de tener un efecto negativo en el nivel de participación electoral cabe buscarlos en las formulaciones teóricas propuestas por Downs (1957). Este autor sugiere que los votantes deciden si participar o no en las elecciones siguiendo un proceso de decisión racional en el que valorarán los beneficios y los costes de tal acción. Los beneficios de ir a votar vendrían representados por la utilidad obtenida si gana el partido preferido por el votante, por los beneficios de representación derivados de participar en el proceso electoral, y por la probabilidad de que acudir a las urnas cambie el resultado de las elecciones. Obviamente la probabilidad de que un solo votante afecte a los resultados de las elecciones es, en la mayor parte de los casos, prácticamente nula. Por este motivo, los beneficios obtenidos por acudir a votar serían, en general, pequeños para la mayoría de votantes. Esto es lo que justifica que algunos costes asociados al hecho de votar, como por ejemplo la incomodidad del desplazamiento, hacer cola para depositar el voto, o soportar las inclemencias meteorológicas, incluso siendo pequeños, pueden ser suficientemente altos como para compensar todas las ganancias obtenidas por participar en el proceso político. Esto haría que algunos votantes prefirieran abstenerse de acudir a las urnas en presencia de dichos costes. O dicho de otra forma, la climatología adversa debería reducir el nivel de participación, ya que supone un aumento del coste de ir a votar.

Muy pocos trabajos han estudiado el tema en profundidad. Knack (1994) fue uno de los primeros en estimar empíricamente el efecto de la climatología en la participación electoral. El trabajo de Knack (1994) combina datos individuales provenientes de encuestas post-electorales en Estados Unidos con datos meteorológicos agregados a nivel de condado. La conclusión alcanzada en este trabajo es que la meteorología apenas tiene efectos en el nivel de participación. Sin embargo los datos utilizados por este autor presentan el problema de que dos de las tres elecciones analizadas en su trabajo son elecciones particularmente secas, lo que dificulta la estimación estadística. En un trabajo posterior, Matsusaka y Palda (1999) llegan a una conclusión similar analizando diversas elecciones en Canadá, y utilizando también datos de encuestas post-electorales. Sachar y Nalebuff (1999) analizan 11 elecciones presidenciales americanas usando datos agregados a nivel de estado en lugar de encuestas individuales. Una de las variables incluidas en su modelo es el nivel de precipitación en la mayor ciudad de cada estado en el día de la elección. Dicha variable tiene un efecto negativo y significativo en la participación electoral. Sin embargo, los resultados no pueden considerarse concluyentes ya que este trabajo presenta como problemas el uso de un nivel de agregación muy alto, y una medida del nivel de lluvia en el estado muy discutible ya que la climatología en el día de las elecciones en la ciudad más grande de un estado no tiene por qué coincidir con la del resto de ciudades, especialmente en aquellos estados con un territorio muy extenso. 
Los resultados poco concluyentes alcanzados por la literatura previa dieron lugar al trabajo de Gómez, Hansford y Krause (2007), que analiza en profundidad el efecto de climatología adversa en el nivel de participación utilizando datos electorales correspondientes a las 14 últimas elecciones americanas desagregados a nivel de condado, y datos sobre el nivel de precipitación correspondientes a 22.000 estaciones meteorológicas repartidas por todo el país. Según este trabajo, el hecho de que llueva en el día de las elecciones reduce la participación electoral en aproximadamente un 1 por 100 por cada pulgada de lluvia, en comparación con un día sin precipitación. Eisinga, Te Grotenbiuis y Pelzer (2011) llegan a resultados similares para el caso holandés utilizando una estrategia de estimación empírica muy parecida.

El presente trabajo pretende complementar y extender los resultados obtenidos por Gómez, Hansford y Krause (2007) y por Eisinga, Te Groenbiuis y Pelzer (2011), aportando la evidencia proporcionada por las últimas elecciones locales celebradas en España.

\section{Modelo econométrico}

La determinación del efecto causal de la meteorología adversa en el nivel de participación electoral requeriría, en condiciones ideales, observar las diferencias en el nivel de participación en municipios en los que llueve en el día de las elecciones y municipios idénticos en los que no llueve en el día de las elecciones. Obviamente, es posible que las condiciones meteorológicas estén correlacionadas con otras características observables del municipio como pueden ser su nivel de renta o desempleo. Por ejemplo, si en general los municipios en los que se observa que ha llovido el día de las elecciones son municipios del norte de España en los que la renta es mayor y el desempleo es menor que en los municipios del sur, en los que no ha llovido, una simple comparación de medias entre el nivel de participación entre los municipios en los que llueve y en los que no, arrojaría resultados que no podrían interpretarse de forma causal. En efecto, la existencia de diferencias en el nivel de participación cabría atribuirlas, al menos en parte, a diferencias en el nivel de renta o desempleo, y no a las distintas condiciones meteorológicas en las que se celebraron las elecciones. Asimismo otras diferencias existentes tanto en términos de variables socio-demográficas como en términos de comportamiento electoral podrían distorsionar los resultados.

Por este motivo, se propone determinar el efecto causal de las condiciones meteorológicas adversas en el nivel de participación mediante la estimación de un modelo de regresión que tenga en cuenta las diferencias observables entre los distintos municipios. En concreto se propone estimar la siguiente ecuación:

$$
\text { Participación }_{i r t}=\alpha+\beta \text { lluvia }_{i t}+\gamma X+\delta_{i}+\eta_{t}+\eta_{t} \theta_{r}+\varepsilon_{i t}
$$


En este modelo la variable Participación hace referencia al porcentaje de electores que votaron en cada elección (e.g. votos válidos/censo) en el municipio $i$, situado en la Comunidad Autónoma $r$, y en las elecciones celebradas en el año $t$; lluvia es el nivel de precipitación en el día de la elección en cada municipio; y $X$ es un vector de variables de control. En cada ecuación se incluye un vector de variables dummy para cada municipio, y para cada elección. Además, se incluye un vector de interacciones Comunidad Autónoma-Elección. Este último término captura efectos que son comunes para los municipios pertenecientes a cada comunidad en cada una de las elecciones.

El modelo especificado captura la mayor parte de factores que pueden distorsionar la relación entre las variables de interés debido a la inclusión, fundamentalmente, de los distintos vectores de variables dummy. En efecto, el vector de efectos fijos para cada municipio captura características de cada municipio que son constantes durante el periodo de estudio. Por otro lado el vector de efectos fijos para cada elección captura las condiciones en las que se celebraron las distintas elecciones, como por ejemplo el clima político a nivel nacional, o la situación económica del país en su conjunto. Por último el vector de interacciones año-región captura características comunes a todos los municipios de una región y en cada año de estudio, como por ejemplo políticas adoptadas por el gobierno regional en cada año analizado. La única fuente de variación no controlada por estos vectores serían shocks idiosincráticos que puede tener cada municipio en cada elección. Para tener en cuenta estos posibles shocks, el vector de variables de control $X$ incluye medidas, tanto lineales como no lineales, que reflejan el tamaño del municipio según su población, y proxies del nivel de renta y desempleo.

En la medida en la que los vectores de variables dummy y el vector de variables socio-demográficas capturan la mayor parte de la variación no aleatoria, la estrategia de identificación del efecto causal en el modelo propuesto consiste, por tanto, en comparar el nivel de participación en municipios en los que llueve con municipios similares en los que no llueve en el día de la elección.

\section{Datos}

En este trabajo se analizan las últimas siete elecciones locales, es decir desde 1987 hasta 2011, ambas incluidas. La estimación de la ecuación [1] requiere datos muy precisos sobre el nivel de precipitación en cada municipio, sobre resultados electorales en dichos municipios, y sobre las variables de control contenidas en el vector $X$.

La información sobre los resultados electorales en las elecciones locales se obtuvo del Ministerio del Interior. Dicha base de datos contiene, para cada municipio, la población, el número de electores, el número de votos válidos, y el número de votos obtenidos por cada candidatura. A partir de esta información se construyó la variable Participación como el porcentaje de votos válidos sobre el total de electores censados en ese municipio. 
La información sobre el nivel de precipitación en cada municipio se obtuvo de la Agencia Estatal de Meteorología. La base de datos de AEMET contiene datos sobre el nivel de precipitación en cada una de las más de 3.000 estaciones meteorológicas que están, o han estado, en funcionamiento en España en alguno de los años de estudio. Dado que el número de municipios en España -8.116- es mucho mayor que el de estaciones meteorológicas, hay muchos municipios en los que no hay ninguna estación en activo. Para solucionar este problema caben dos alternativas. La primera de ellas consistiría en imputar el nivel de precipitación de los municipios sobre los que no se dispone de información precisa, por ejemplo utilizando como proxy el nivel de precipitación de municipios cercanos. Esta opción presenta el problema de que introduce un error de medida en la variable lluvia. Por este motivo, en este trabajo se optó por una segunda opción, que es utilizar en la estimación únicamente aquellos municipios en los que existe una estación meteorológica en funcionamiento en, al menos, una de las elecciones locales analizadas. Por otro lado, en algunos municipios hay más de una estación meteorológica en funcionamiento en los años analizados. Esto ocurre sobre todo en los municipios grandes como Madrid o Barcelona. En estos casos se optó por utilizar como medida de precipitación de ese municipio la media de precipitación de todas las estaciones meteorológicas incluidas dentro del término municipal. En total se incluyeron 2.970 municipios distintos, y un total de 14.911 observaciones.

Por lo que se refiere a las variables de control, se incluyeron tres: población total del municipio, nivel de desempleo y una proxy del nivel de renta. La población se obtuvo de la base de datos de resultados electorales del Ministerio del Interior. El desempleo y la proxy del nivel de renta se obtuvieron del Anuario Económico de La Caixa. Esta base de datos contiene información a nivel municipal sobre paro registrado y sobre otras variables relacionadas con la situación económica del municipio. La proxy de renta que se utilizó en este trabajo es una variable que la base de datos de la Caixa denomina Cuota de mercado del municipio. Dicha variable es un índice que expresa la capacidad de compra comparativa de cada municipio en cada año analizado. Se construye utilizando el promedio de otros índices contenidos en el Anuario de La Caixa, y que tienen en cuenta los siguientes indicadores: población, número de teléfonos fijos (de uso doméstico), automóviles, camiones (camiones y furgonetas), oficinas bancarias, y actividades comerciales minoristas. En general esta variable pretende medir la capacidad de consumo de cada municipio.

La base de datos de La Caixa sólo contiene información para municipios con más de mil habitantes, que son aproximadamente un tercio del total de municipios españoles. La inclusión en la estimación de las variables contenidas en esta base de datos reduce la muestra a 4.996 observaciones en las especificaciones que las contienen. Sin embargo esto no es un problema grave ya que distintas pruebas de robustez de los resultados confirman que el efecto estimado de la lluvia en el nivel de participación es cualitativamente bastante similar en las estimaciones que incluyen las variables socio-económicas y en las que no las incluyen.

Por último cabe destacar que se utilizaron varias medidas del nivel de precipitación y todas ellas arrojaron resultados cualitativamente similares. La primera de estas 
variables es el nivel de precipitación en milímetros ${ }^{4}$. Esta variable presenta el problema de que no tiene en cuenta que el mismo nivel de lluvia debería tener efectos muy distintos dependiendo de si el municipio está más o menos acostumbrado a la meteorología adversa. Por ejemplo, un cierto nivel de lluvia es probable que tenga mayor efecto en la participación en municipios no acostumbrados a la lluvia (por ejemplo municipios de Murcia o Almería), mientras que en Galicia dicho nivel de lluvia tendrá un efecto previsiblemente menor ya que los electores de esta región están más acostumbrados a los días lluviosos. Para resolver este problema se estimó el modelo en una especificación alternativa utilizando como variable el nivel de precipitación en desviaciones respecto a la media histórica de precipitación en ese municipio y en ese día del año $0^{5}$. Para construir la media en cada municipio se utilizaron datos de los últimos 40 años proporcionados por AEMET. Finalmente, también se estimó el modelo definiendo la variable Dummy lluvia simplemente como una variable dicotómica a la que se le dio valor 1 en los municipios en los que llovió en el día de las elecciones y 0 en los que no llovió. Esta última variable captura simplemente el efecto medio de un día lluvioso en el nivel de participación electoral frente a municipios similares en los que no llovió.

\section{Resultados}

En el Cuadro 1 se presentan los estadísticos descriptivos de los resultados electorales de cada una de estas elecciones así como de las condiciones meteorológicas en las que se celebraron. Como puede observarse, el periodo analizado comprende elecciones celebradas bajo distintas condiciones meteorológicas, lo que resulta conveniente desde el punto de vista estadístico. Por ejemplo, las elecciones de 1991 se celebraron en condiciones muy secas (menos de un 1 por 100 de municipios sufrieron precipitaciones durante las elecciones), mientras que en las elecciones de 2007 llovió en más de un tercio de los municipios españoles. Por lo que respecta a los resultados electorales, la tabla muestra la preponderancia de los dos principales partidos, PP y PSOE, que obtienen de forma conjunta aproximadamente dos tercios del total de votos válidos en cada una de las elecciones.

El Cuadro 2 muestra los principales resultados de este trabajo. En esta tabla cada columna corresponde a diferentes especificaciones de la ecuación [1]. En las tres primeras columnas se estima el modelo utilizando como variable explicativa el nivel de lluvia en cada localidad en el día de las elecciones, en milímetros. En las tres siguientes columnas se utiliza una dummy que adopta el valor 1 si llovió en el municipio en el día de las elecciones y 0 si no llovió. Como puede observarse en las tres primeras columnas, el nivel de precipitación tiene un efecto estadísticamente

${ }^{4}$ El milímetro lineal es una medida utilizada habitualmente en Meteorología para evaluar la cantidad de lluvia. Un milímetro equivale a un litro por metro cuadrado.

${ }^{5}$ Los resultados de esta estimación están disponibles previa petición al autor. 


\section{CUADRO 1}

RESULTADOS ELECTORALES Y CONDICIONES METEOROLÓGICAS EN LAS ELECCIONES LOCALES ESPAÑOLAS

\begin{tabular}{|l|c|c|c|c|c|c|c|}
\hline & $\mathbf{1 9 8 7}$ & $\mathbf{1 9 9 1}$ & $\mathbf{1 9 9 5}$ & $\mathbf{1 9 9 9}$ & $\mathbf{2 0 0 3}$ & $\mathbf{2 0 0 7}$ & $\mathbf{2 0 1 1}$ \\
\hline Fecha de elección & 10 junio & 26 mayo & 28 mayo & 13 junio & 25 mayo & 27 mayo & 22 mayo \\
\hline $\begin{array}{l}\text { Resultados electorales } \\
\text { Porcentaje de participación }\end{array}$ & 69,42 & 62,78 & 69,87 & 63,99 & 67,67 & 63,97 & 66,16 \\
\hline Porcentaje de voto PSOE & 37,08 & 38,34 & 30,84 & 34,26 & 34,83 & 34,92 & 27,79 \\
\hline Porcentaje de voto PP & 20,38 & 25,34 & 35,27 & 34,44 & 34,29 & 35,62 & 37,54 \\
\hline Porcentaje de voto (otros) & 42,54 & 36,32 & 33,89 & 31,3 & 30,88 & 29,46 & 34,67 \\
\hline $\begin{array}{l}\text { Condiciones meteorológicas } \\
\text { Lluvia (en mm) }\end{array}$ & 0,638 & 0,012 & 0,058 & 2,454 & 0,759 & 1,248 & 0,499 \\
\hline $\begin{array}{l}\text { Porcentaje de municipios } \\
\text { que experimentan lluvia }\end{array}$ & 14,16 & 0,6 & 1,92 & 33,10 & 11,34 & 31,73 & 13,88 \\
\hline
\end{tabular}

significativo en el nivel de participación electoral en las elecciones locales españolas. El coeficiente de la variable lluvia es siempre significativo y tiene una magnitud de $-0,038$ en la estimación de la primera columna. La magnitud del coeficiente es todavía mayor, $-0,06$, en la estimación de la columna 3 , que incluye como controles las proxies del nivel de renta y del nivel de desempleo. Estas tres columnas muestran que la meteorología adversa provoca una bajada en el nivel de participación electoral en aquellos municipios que la sufren, en comparación con municipios similares en los que no llovió en el día de las elecciones. Esta conclusión se confirma en las regresiones mostradas en las columnas 4, 5 y 6 . La dummy que captura si llovió o no en el día de las elecciones muestra que el nivel de participación se redujo en aproximadamente medio punto porcentual en aquellos municipios que sufrieron la presencia de precipitación durante la jornada electoral frente a aquellos municipios en los que la lluvia no hizo acto de presencia.

Una vez comprobado que las malas condiciones meteorológicas aumentan la abstención, resulta interesante comprobar si la meteorología es susceptible de alterar los resultados electorales. Si la lluvia afectase a los votantes de todos los partidos por igual, no tendría efectos sobre el resultado de las elecciones, ya que aunque la participación se redujera, el porcentaje de voto de cada partido quedaría más o menos constante. Sin embargo, según apuntan diversos trabajos, parece razonable pensar que serán los partidos de izquierdas los más perjudicados por la bajada en la participación si las elecciones se celebran en condiciones meteorológicas adversas. La razón sería que las personas de renta baja son más susceptibles de verse afectadas por el mayor coste que supone ir a votar en condiciones meteorológicas adversas ya que, por ejemplo, no disponen de vehículos para desplazarse, o viven más alejadas del centro del municipio, donde suelen estar ubicados los colegios electorales. 
METEOROLOGÍA Y RESULTADOS ELECTORALES EN LAS ELECCIONES LOCALES ESPAÑOLAS 151

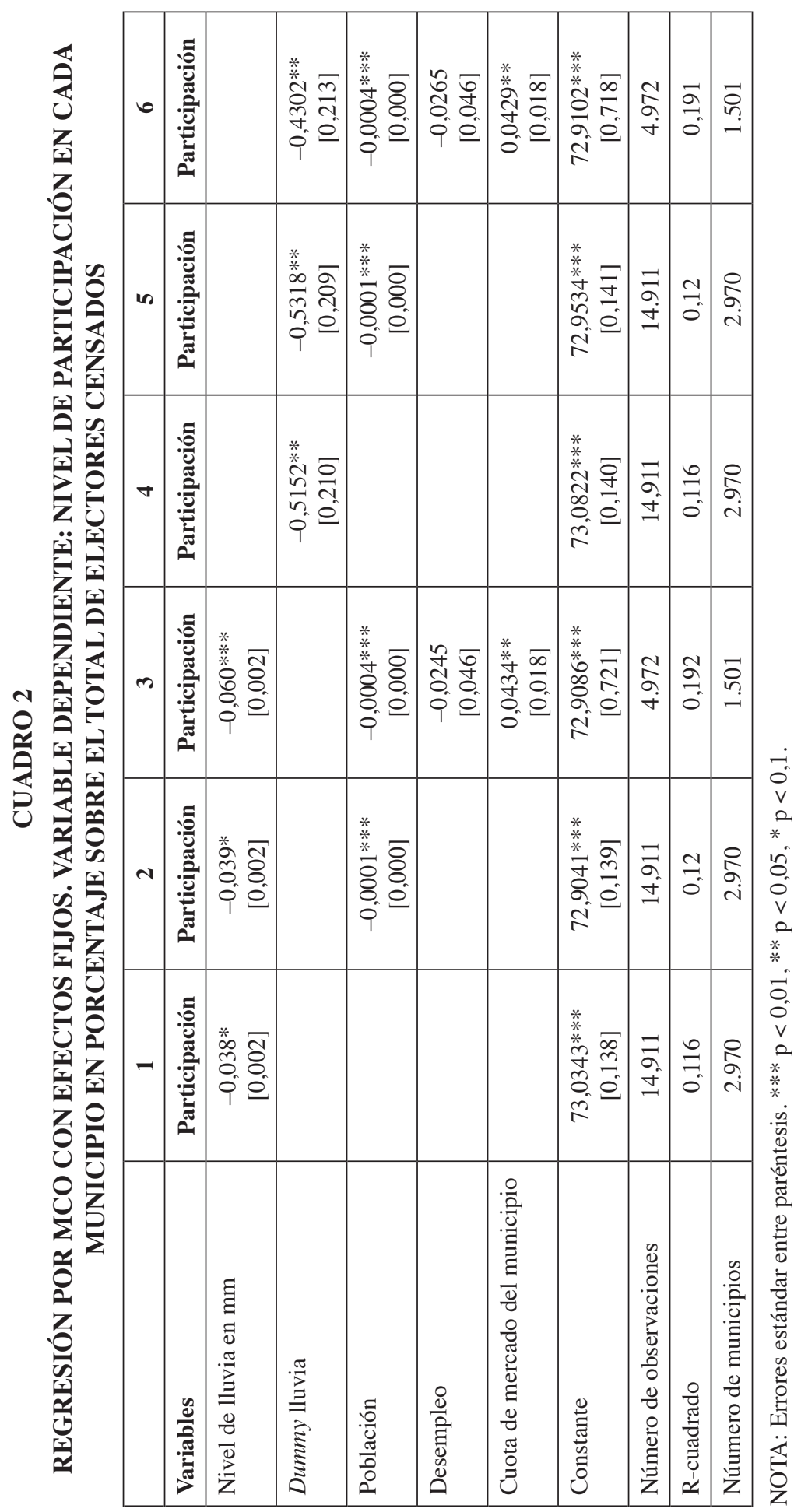


Para estudiar si la meteorología adversa tiene efectos sobre los resultados que obtiene cada partido político, en el Cuadro 3 se estima el mismo modelo que en el Cuadro 2 pero utilizando como variable dependiente el porcentaje de voto del Partido Popular (columnas 1 y 2) y el porcentaje de voto del Partido Socialista (columnas 3 y 4). Como puede comprobarse, la meteorología adversa parece resultar beneficiosa para el Partido Popular y resultaría perjudicial para el Partido Socialista. El coeficiente de las dos primeras columnas muestra que por cada milímetro de lluvia adicional en unas elecciones, el PP vería incrementado su porcentaje de voto entre 0,04 (columna 1) y 0,13 (columna 2) puntos porcentuales. Si tenemos en cuenta que la lluvia es un fenómeno localmente concentrado, y que en nuestra base de datos en los municipios en los que llueve la media de precipitación diaria es de 5,4 mm, esto implica que comparado con un municipio en el que no llueve el PP incrementa su cuota de voto entre $0,25(0,04 \times 5,4)$ y $0,70(0,13 \times 5,4)$ puntos porcentuales. Igualmente, el Cuadro 3 muestra que el PSOE resultaría en general perjudicado por el aumento de la precipitación en aproximadamente la misma medida en la que el PP resulta beneficiado. Los resultados del Cuadro 3, sin embargo, deben tomarse con cautela ya que, por un lado, el coeficiente relevante no es significativo en algunas de las estimaciones y, por otro lado, dicho coeficiente cambia en magnitud al añadir las variables Desempleo y Cuota de mercado.

\section{CUADRO 3}

REGRESIÓN POR MCO CON EFECTOS FIJOS. VARIABLE DEPENDIENTE: CUOTA DE VOTO DE PP O PSOE COMO PORCENTAJE DEL TOTAL DE VOTOS VÁLIDOS

\begin{tabular}{|c|c|c|c|c|}
\hline & 1 & 2 & 3 & 4 \\
\hline Variables & $\begin{array}{c}\text { Cuota de voto } \\
\text { PP }\end{array}$ & $\begin{array}{c}\text { Cuota de voto } \\
\text { PP }\end{array}$ & $\begin{array}{c}\text { Cuota de voto } \\
\text { PSOE }\end{array}$ & $\begin{array}{c}\text { Cuota de voto } \\
\text { PSOE }\end{array}$ \\
\hline Nivel de lluvia en mm & $\begin{array}{l}0,043 \\
{[0,04]}\end{array}$ & $\begin{array}{c}0,131 * * * \\
{[0,04]} \\
\end{array}$ & $\begin{array}{c}-0,046 \\
{[0,004]}\end{array}$ & $\begin{array}{c}-0,107 * * \\
{[0,005]}\end{array}$ \\
\hline Población & $\begin{array}{c}0,0001 * * * \\
{[0,000]}\end{array}$ & $\begin{array}{l}0,0002 \\
{[0,000]}\end{array}$ & $\begin{array}{l}0,00009 \\
{[0,000]}\end{array}$ & $\begin{array}{l}0,00009 \\
{[0,000]}\end{array}$ \\
\hline Desempleo & & $\begin{array}{c}0,2353 * * \\
{[0,094]}\end{array}$ & & $\begin{array}{r}-0,0883 \\
{[0,103]}\end{array}$ \\
\hline Cuota de mercado del municipio & & $\begin{array}{c}-0,0196 \\
{[0,038]}\end{array}$ & & $\begin{array}{c}-0,0193 \\
{[0,041]}\end{array}$ \\
\hline Constante & $\begin{array}{c}31.2125^{* * * *} \\
{[0,327]}\end{array}$ & $\begin{array}{c}27,7402 * * * \\
{[1,498]}\end{array}$ & $\begin{array}{c}42,0277 * * * \\
{[0,2645]}\end{array}$ & $\begin{array}{c}38,5122 * * * \\
{[1,616]}\end{array}$ \\
\hline Número de observaciones & 13.608 & 4.924 & 13.813 & 4.882 \\
\hline R-cuadrado & 0,145 & 0,173 & 0,085 & 0,092 \\
\hline Número de municipios & 2.873 & 1.496 & 2.885 & 1.496 \\
\hline
\end{tabular}

NOTA: Errores estándar entre paréntesis. *** $\mathrm{p}<0,01, * * \mathrm{p}<0,05, * \mathrm{p}<0,1$. 
Pese a la cautela anterior, con objeto de dar una idea de lo que implican cuantitativamente los resultados obtenidos, tomemos como referencia las estimaciones más precisas del Cuadro 3, y pongamos como ejemplo las últimas elecciones locales. En las elecciones de 2011 hubo un 5 por 100 de municipios en los que, según nuestra base de datos, la diferencia de voto entre PP y PSOE fue menor a 1 punto porcentual (406 municipios). Dado que en estas elecciones llovió en aproximadamente en un 14 por 100 de estos municipios, esto significa que en aproximadamente 57 municipios, el resultado electoral hubiera sido distinto si no hubiese llovido. Aunque a nivel nacional este número pueda parecer pequeño (sólo 57 municipios de un total de 8.116 experimentarían un cambio de resultado electoral), obviamente en unas elecciones más reñidas y más lluviosas, la magnitud sería mayor. En definitiva, nuestros resultados muestran que si bien la magnitud del efecto de la lluvia en los resultados electorales es pequeña, es susceptible de cambiar el resultado electoral en algunos municipios.

\section{Conclusión}

En este trabajo se han utilizado datos de las elecciones locales españolas para contrastar una de las implicaciones más claras de la teoría racional del voto: los votantes llevan a cabo una evaluación de los beneficios y los costes de ir a votar y deciden acudir a las urnas si los beneficios superan a los costes. Si aumentan los costes de ir a votar, ceteris paribus, deberíamos observar un menor nivel de participación. Como medida del coste de ir a votar, en este trabajo se ha utilizado el nivel de lluvia en el día de las elecciones. Nuestras estimaciones muestran que en aquellos municipios en los que llovió en el día de las elecciones el nivel de participación fue aproximadamente medio punto porcentual menor que en municipios similares que en los que no llovió el día de las elecciones.

Por otro lado, en este trabajo también se ha analizado si la lluvia en el día de las elecciones afecta de forma distinta a los votantes de distintos partidos políticos en las elecciones locales españolas. Se ha comprobado que el PP resultaría beneficiado por las malas condiciones meteorológicas mientras que los partidos de izquierdas resultarían perjudicados. Este resultado concuerda con las teorías que argumentan que los votantes conservadores se ven menos afectados por el aumento de los costes de ir a votar, y es además consistente con las teorías que desde la economía política argumentan que los votantes de derechas son más expresivos que los de izquierdas, y por tanto más propensos a ir a votar.

Por último cabe reseñar que este trabajo supone únicamente una primera aproximación al estudio de los efectos de la meteorología adversa en España. Los resultados obtenidos podrían completarse en el futuro con el estudio de las elecciones generales o autonómicas, y con un análisis econométrico más detallado que tenga en cuenta no sólo el efecto de la lluvia, sino la temperatura u otras variables relevantes para determinar la desutilidad que le supone al votante acercarse a las urnas en un 
día concreto. Además, en el futuro esta línea de investigación podría extenderse para estudiar con mayor precisión el efecto de la abstención en los resultados electorales o en otras variables relevantes.

\section{Referencias bibliográficas}

[1] DOWNS, A. (1957): An economic theory of democracy, Harper and Row, New York.

[2] KNACK, S. (1994): «Does rain help the republicans? Theory and Theory and evidence on turnout and the vote», Public Choice, 79, 187-209.

[3] MATSUSAKA, J. G. y PALDA, F. (1999): «Voter turnout: How much can we explain?», Public Choice 98, 431-446.

[4] SHACHAR, R. y NALEBUFF, B (1999): «Follow the leader. Theory and evidence on political participation», American Economic Review, 89, 525-547.

[5] GOMEZ, B. T.; HANSFORD, T. G. y KRAUSE, G. A. (.2007): «The republicans should pray for rain. Weather, turnout, and voting in U.S. presidential elections», Journal of Politics, 69, 649-663.

[6] EISINGA, R.; TE GROTENHUIS, M. y PELZER, B. (2011): «Weather conditions and voter turnout in Dutch national parliament elections, 1971-2010», International Journal of Biometeorology. doi:10.1007/s00484011-0477-7. 